\title{
Home glucose meters: How accurate should they be to avoid dysglycemia in patients using carbohydrate counting?
}

Cite as: AIP Conference Proceedings 2116, 250010 (2019); https://doi.org/10.1063/1.5114250

Published Online: 24 July 2019

Carlos Abreu (D), Francisco Miranda (D), Anna Dabrowska, and Paula Felgueiras (D)

\section{Conference Proceedings}

Get $30 \%$ off all print proceedings!

\section{Enter Promotion Code PDF-30 at checkout}

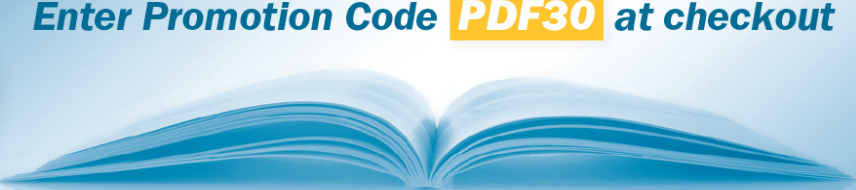




\title{
Home Glucose Meters: How Accurate Should They Be to Avoid Dysglycemia in Patients Using Carbohydrate Counting?
}

\author{
Carlos Abreu ${ }^{1,2, a)}$, Francisco Miranda ${ }^{1,3}$, Anna Dabrowska ${ }^{1,4}$ and Paula \\ Felgueiras ${ }^{1,5}$ \\ ${ }^{1}$ Instituto Politécnico de Viana do Castelo, Viana do Castelo, Portugal \\ ${ }^{2}$ CMEMS-UMinho, Universidade do Minho, Braga, Portugal \\ ${ }^{3}$ CIDMA, Universidade de Aveiro, Aveiro, Portugal \\ ${ }^{4}$ Bialystok University of Technology, Bialystok, Poland \\ ${ }^{5}$ Unidade Local de Saúde do Alto Minho, Hospital de Santa Luzia, Viana do Castelo, Portugal \\ ${ }^{a)}$ Corresponding author: cabreu@estg.ipvc.pt
}

\begin{abstract}
Accurate self-monitoring of blood glucose is the key to an effective and safe intensive insulin therapy. Indeed, most insulin dosing decisions are made based on the blood glucose values obtained from home glucose meters, in particular for those using diet planning and carbohydrate counting. Patients on that therapeutic regimen depend not only on their ability to accurately estimate the carbohydrate content of each meal but also on the accuracy of the glucose meter being used. Therefore, in order to avoid postprandial hypoglycemia and hyperglycemia, it is of great importance to realize how important is the accuracy of blood glucose meters according to the particular characteristics of each patient. In this regard, we propose an analytic method to find the limits of the blood glucose meters accuracy according to the insulin-to-carb ratio, the insulin sensitivity factor and the ability of each patient to estimate the carbohydrate content of each meal.
\end{abstract}

\section{INTRODUCTION}

Self-Monitoring of Blood Glucose (SMBG) is the mainstay of proper diabetes management, supporting tight blood glucose control in patients on intensive insulin regimens, e.g., patients using diet planning and carbohydrate counting. However, to be useful and safe SMBG needs to be accurate. The accuracy of SMBG systems relies on several factors, being the accuracy of the blood glucose meters one of the most important [1]. As reported by Ekhlaspour et al in [2], the accuracy of commercially available blood glucose meters varies significantly. Regarding this study, 17 glucose meters were tested and it was found that their mean absolute relative difference (MARD) range from 5.6\% to 20.8\%.

Inaccurate blood glucose measurements may lead to incorrect dosing calculations which may result in dangerous variations of insulin doses and negatively influence glycemic control $[1,3]$. Indeed, Campos-Náñez et al conducted a 30-day in-silico study and found that blood meters accuracy clearly affect the glycemic control in type 1 diabetes patients. Moreover, the study shows that the meters' accuracy has a significant impact not only on the occurrence of severe hypoglycemic events but also on long-term average glycemia (HbA1c) [4].

In this context, it is of great importance to choose the correct blood glucose meter taking into account the particular characteristics and glycemic goals of each patient. Therefore, we propose an analytic method to find the limits of the blood glucose meters accuracy that considers the ability of each patient to estimate the carbohydrate content of each meal ${ }^{1}$ along with its' insulin-to-carb ratio and insulin sensitivity factor.

\footnotetext{
${ }^{1}$ The ability of each patient to accurately estimate the carbohydrate content of each meal could be quantified considering the average absolute error committed by the patient while evaluating a set of $\mathrm{N}$ meals, by: $\overline{\Delta C H O}=\sum_{i=1}^{N}\left|C H O_{\text {correct }}^{i}-C H O_{\text {estimate }}^{i}\right| / N$, where the $C H O_{\text {correct }}^{i}$ and the $\mathrm{CHO}_{\text {estimate }}^{i}$ are, respectively, the correct and estimate values of the carbohydrate content of $i$-meal, $i=1, \ldots, N$.
}

International Conference of Numerical Analysis and Applied Mathematics (ICNAAM 2018)

AIP Conf. Proc. 2116, 250010-1-250010-4; https://doi.org/10.1063/1.5114250

Published by AIP Publishing. 978-0-7354-1854-7/ $\$ 30.00$ 


\section{MATHEMATICAL METHODS}

The equation

$$
B=\left(\frac{C H O}{I C R}+\frac{G-G_{T}}{I S F}\right) K-I O B
$$

allows patients using carbohydrate counting to calculate the insulin bolus (i.e., prandial and correctional insulin) for each meal, according to the carbohydrates planned to be consumed in that meal $(\mathrm{CHO})$, the insulin-to-carb ratio $(I C R)$, the preprandial blood glucose target $\left(G_{T}\right)$, the preprandial blood glucose value $(G)$, the insulin sensitivity factor $(I S F)$, the physiologic status of the patient represented by $K$, and the insulin remaining active from the last bolus ( $I O B$, Insulin-on-Board) [5, 6]. In the subsequent analysis, we will consider $K=1$ (i.e., the impact of illness, physical activity or medication on insulin and glucose metabolism of patients will not be addressed in this study) and $I O B=0$ (i.e., it will be considered that the time between meals is larger than the duration of the insulin action, and there is no insulin stacking). Moreover, we also assume that patient's ICR and $I S F$ are physiologically appropriate.

Let's consider $C H O=C \hat{H} O \pm \triangle C H O$ and $G=\hat{G} \pm \Delta G$ to be variables of Equation 1, where $C \hat{H} O$ is an estimate of $C H O$ with an absolute error $\Delta C H O>0$, and $\hat{G}$ is an estimate of $G$ with an absolute error $\Delta G>0$. The remaining variables of Equation 1 are considered to be exact values. In such conditions, the absolute error of $B$ is $\Delta B=|B-\hat{B}|$, where: $B=C H O / I C R+\left(G-G_{T}\right) / I S F$ and $\hat{B}=C \hat{H} O / I C R+\left(\hat{G}-G_{T}\right) / I S F$ (n.b., ICR and $I S F$ are positive values). Therefore, we have:

$$
\Delta B=\left|\frac{C H O-C \hat{H} O}{I C R}+\frac{G-\hat{G}}{I S F}\right| \leq \frac{\Delta C H O}{I C R}+\frac{\Delta G}{I S F} .
$$

The bolus' absolute error, $\Delta B$, will perform as an unplanned and inappropriate correction bolus, with the consequent undesired effect on the patient's postprandial glycemia $\left(G_{\text {postprandial }}\right)$ given by:

$$
\Delta G_{\text {postprandial }}=\Delta B \cdot I S F \leq \frac{I S F}{I C R} \Delta C H O+\Delta G,
$$

where $\Delta G_{\text {postprandial }}$ is the variation on the patient postprandial blood glucose.

By denoting the hyperglycemia and hypoglycemia limits as $G_{H y p e r}$ and $G_{H y p o}$, respectively, it is possible to conclude that the maximum value of $\Delta G_{\text {postprandial }}$ allowed, in order to avoid hyperglycemia and hypoglycemia episodes, is given by $\min \left\{G_{\text {Hyper }}-G_{T}, G_{T}-G_{\text {Hypo }}\right\}$ (n.b., the function $\min \{\cdot\}$ returns the minimum value of a set of elements) and, therefore, it is sufficient to satisfy the following condition to avoid them:

$$
\Delta C H O \leq \frac{I C R}{I S F}\left(\min \left\{G_{H y p e r}-G_{T}, G_{T}-G_{H y p o}\right\}-\Delta G\right) .
$$

Equation 2 shows that the sufficient limits of accurate carbohydrate counting, in order to avoid dysglycemia, depend not only on each patient data but also on the accuracy of the blood glucose meter being used. Therefore, taking into account the ability of each patient to correctly estimate the carbohydrate content of each meal, along with it's $I S F, I C R$, and glycemic targets, the blood glucose meter absolute error, $\Delta G$, should satisfy the following conditions:

$$
\begin{gathered}
\Delta G \leq \min \left\{G_{H y p e r}-G_{T}, G_{T}-G_{H y p o}\right\}-\frac{I S F}{I C R} \overline{\Delta C H O} \\
\text { and } \\
\overline{\Delta C H O} \leq \frac{I C R}{I S F}\left(\min \left\{G_{H y p e r}-G_{T}, G_{T}-G_{H y p o}\right\}-\Delta G\right) .
\end{gathered}
$$

\section{DISCUSSION}

To assess and discuss the implications of the proposed method let's consider a hypothetical patient of $75 \mathrm{~kg}$ body weight, consuming a total of $50 \mathrm{U}$ of insulin each day, having a preprandial glucose target of $G_{T}=110 \mathrm{mg} / \mathrm{dL}$, and the following glycemic thresholds for hyperglycemia and hypoglycemia, respectively: $G_{\text {Hyper }}=150 \mathrm{mg} / \mathrm{dL}, G_{\text {Hypo }}=$ $70 \mathrm{mg} / \mathrm{dL}$. Suppose, without loss of generality, that the $I C R$ and $I S F$ for this patient are calculated according to the recommendations of the American Association of Clinical Endocrinologists/American College of Endocrinology 
(AACE/ACE) [7], using the following rules: $I C R=450 / T D D$ and $I S F=1700 / T D D$, where TDD is the Total Daily Dose of insulin that, in this case, is equal to $50 \mathrm{U}$. Therefore, under these assumptions, the patient's $I C R$ and $I S F$ are $9 \mathrm{~g} / \mathrm{U}$ and $34 \mathrm{mg} / \mathrm{dL} / \mathrm{U}$, respectively. Finally, this patient is able to estimate the carbohydrate content of each meal with an average absolute error of $10 \mathrm{~g}$ (i.e., $\overline{\triangle C H O}=10 \mathrm{~g}$ ).

According to Equation 2, the maximum absolute error allowed while estimating the carbohydrate content of each meal depends on the meter accuracy and on the patient data, as shown Figure 1. Let us consider first the use of an ideal blood glucose meter, i.e., having no error. In that case, the maximum admissible absolute error while estimating the carbohydrates is given by $\Delta C H O_{\max }=9 \times 40 / 34 \approx 10.59 \mathrm{~g}$. Hence, since $\overline{\Delta C H O} \leq \Delta C H O_{\max }$, the risk of the patient facing dysglycemic events is reduced. However, such an ideal meter does not exist. Moreover, the accuracy of the blood glucose meters available on the market varies significantly, as can be seen in Table 1.

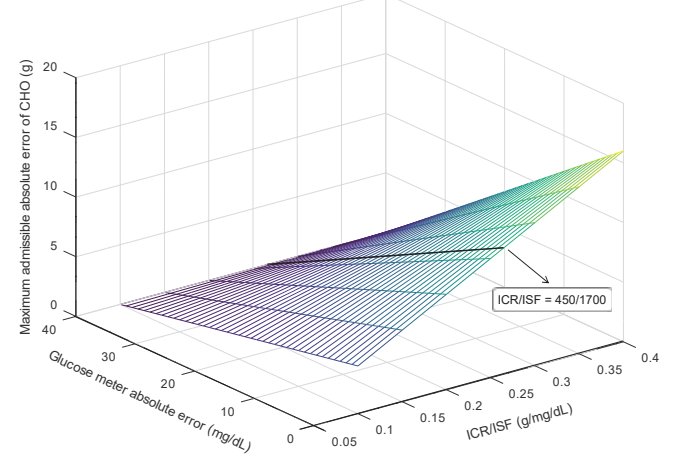

a)

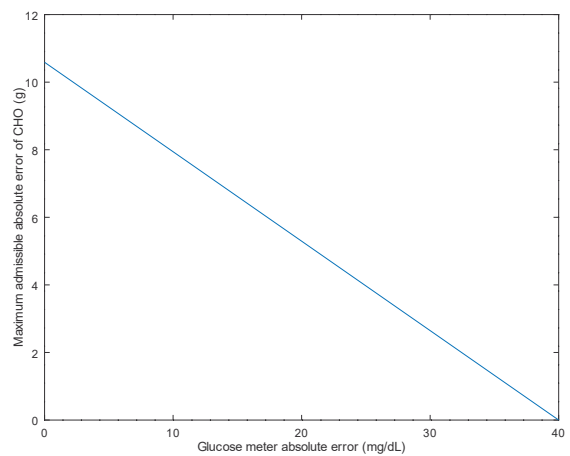

b)

FIGURE 1. a) The maximum admissible absolute error of $C H O$ as a function of the glucose meter accuracy and the $I C R / I S F$ ratio; b) The maximum admissible absolute error of $C H O$ as a function of the glucose meter accuracy for $I C R / I S F=450 / 1700$.

Taking into account the accuracy of the blood glucose meters, the value of $\triangle C H O_{\max }$ could be significantly smaller. Table 1 shows the value of $\triangle C H O_{\max }$ for several blood glucose meters and, makes clear that none of them fits into the diabetes management strategy of this patient. Therefore, this patient must improve their carbohydrates counting skills or change their glycemic targets. As an example, if the patient glycemic goals change to $G_{T}=$ $120 \mathrm{mg} / \mathrm{dL}, G_{\text {Hyper }}=170 \mathrm{mg} / \mathrm{dL}$, and $G_{\text {Hypo }}=70 \mathrm{mg} / \mathrm{dL}$, it will be safe to use any of the first four blood glucose meters listed in Table 1, under the range $20 \leq B S<180$.

Therefore, the accuracy of the blood glucose meters is a determinant factor for a proper glycemic control and must be taken into account while fitting the diabetes management strategy of each patient.

\section{CONCLUSION}

Patients with diabetes make their insulin dosing decisions based on the glycemic values obtained from home glucose meters. Indeed, patients on intensive insulin regimens, particularly those using meal planning and carbohydrate counting, heavily depend not only on their ability to accurately estimate the carbohydrate content of each meal but also on the accuracy of the blood glucose meters to achieve a rigorous glycemic control. Therefore, to avoid dysglycemic events, it is fundamental to understand the impact of the blood glucose meters accuracy on the insulin bolus. To that end, we propose an analytic method to find the limits of the blood glucose meters accuracy according to the insulin-to-carb ratio, the insulin sensitivity factor and the ability of each patient to estimate the carbohydrate content of each meal. By using the proposed method, healthcare professionals are able to make informed decisions about the best blood glucose meter for each patient according to their particular characteristics. Moreover, this method allows healthcare professionals to optimize the glycemic goals of each patient according to their characteristics and meter accuracy in order to lessen the risk of hypoglycemic and hyperglycemic episodes. 
TABLE 1. The maximum of the glucose meter absolute error $\left(\Delta G_{\max }\right)$, and the maximum admissible absolute error of carbohydrate counting $\left(\triangle C H O_{\max }\right)$ for each glucose meter. The value of $\Delta G_{\max }$ was estimated according to the data reported in Table 2 of [2], for different glucose ranges as: $\Delta G_{\max } \approx M A R D \times \max \{B S$ in the range $\} / 100$ (n.b., $B S$ stands for Blood Sugar). On its turn, $\triangle C H O_{\max }$ was calculated according to Equation 2, taking into account the $\Delta G_{\max }$ of each glucose meter, and the specific characteristics of the patient being considered.

\begin{tabular}{|c|c|c|c|c|c|c|}
\hline \multirow{3}{*}{ Blood Glucose Meter } & \multicolumn{6}{|c|}{ Blood Sugar Ranges (mg/dL) } \\
\hline & \multicolumn{2}{|c|}{$20 \leq B S<70$} & \multicolumn{2}{|c|}{$70 \leq B S<180$} & \multicolumn{2}{|c|}{$180 \leq B S<440$} \\
\hline & $\Delta G_{\max }$ & $\triangle C H O_{\max }$ & $\Delta G_{\max }$ & $\triangle C H O_{\max }$ & $\Delta G_{\max }$ & $\triangle C H O_{\max }$ \\
\hline \# 1 Contour Next & 6.23 & 8.94 & 8.64 & 8.30 & 23.76 & 4.30 \\
\hline \# 2 StatStrip Xpress & 7.77 & 8.53 & 8.82 & 8.25 & 26.40 & 3.60 \\
\hline \# 3 OneTouch VeriolQ & 6.93 & 8.75 & 11.34 & 7.59 & 29.92 & 2.67 \\
\hline \# 4 Accu-Chek Nano & 8.12 & 8.44 & 12.06 & 7.40 & 29.92 & 2.67 \\
\hline \# 5 FreeStyle Freedom Lite & 10.78 & 7.73 & 13.14 & 7.11 & 27.28 & 3.37 \\
\hline \# 6 Accu-Chek Aviva Plus & 8.47 & 8.35 & 13.14 & 7.11 & 30.36 & 2.55 \\
\hline \# 7 FreeStyle Lite & 11.62 & 7.51 & 14.22 & 6.82 & 29.92 & 2.67 \\
\hline \# 8 Nova Max & 18.90 & 5.59 & 16.02 & 6.35 & 30.36 & 2.55 \\
\hline \# 9 TRUEresult & 9.24 & 8.14 & 23.04 & 4.49 & 57.64 & $*$ \\
\hline \# 10 HemoCue Glucose 201 & 13.93 & 6.90 & 20.88 & 5.06 & 55.44 & $*$ \\
\hline \# 11 OneTouch Ultra2 & 21.21 & 4.97 & 28.26 & 3.11 & 42.68 & $*$ \\
\hline \# 12 ReliOn Prime & 11.34 & 7.59 & 20.52 & 5.16 & 66.44 & $*$ \\
\hline \# 13 BREEZE R2 & 17.99 & 5.83 & 23.94 & 4.25 & 65.56 & $*$ \\
\hline \# 14 ReliOn Micro & 20.93 & 5.05 & 25.56 & 3.82 & 62.04 & $*$ \\
\hline \# 15 AgaMatrix PRESTO & 27.44 & 3.32 & 38.16 & 0.49 & 43.12 & $*$ \\
\hline \# 16 AgaMatrix JAZZ & 26.60 & 3.55 & 40.14 & $*$ & 46.20 & $*$ \\
\hline \# 17 SideKick & 22.19 & 4.71 & 30.06 & 2.63 & 89.76 & $*$ \\
\hline
\end{tabular}

\section{ACKNOWLEDGMENTS}

Carlos Abreu and Paula Felgueiras have received a research grant from Ascensia Diabetes Care Portugal under the project: Health Equity for Diabetics: An Intervention for Adults With Type 2 Diabetes in the North Region of Portugal.

This work was supported by Portuguese funds through the CIDMA - Center for Research and Development in Mathematics and Applications, and the Portuguese Foundation for Science and Technology, within project UID/MAT/04106/2013.

\section{REFERENCES}

[1] Garg S. K. and Hirsch I. B. Self-monitoring of blood glucose. Diabetes Technology E Therapeutics, 20(S1):S-3-S-12, 2018.

[2] Ekhlaspour L., Mondesir D., Lautsch N., Balliro C., Hillard M., Magyar K., Radocchia L. G., Esmaeili A., Sinha M., and Russell S. J. Comparative accuracy of 17 point-of-care glucose meters. Journal of Diabetes Science and Technology, 11(3):558-566, 2017.

[3] Jendrike N., Baumstark A., and Pleus S., et al. Accuracy of five systems for self-monitoring of blood glucose in the hands of adult lay-users and professionals applying iso 15197: 2013 accuracy criteria and potential insulin dosing errors. Current Medical Research and Opinion, 0(ja):1-21, 2018.

[4] Campos-Náñez E., Fortwaengler K., and Breton M. D. Clinical impact of blood glucose monitoring accuracy: An in-silico study. Journal of Diabetes Science and Technology, 11:1187-1195, 2017.

[5] Schmidt S. and Nørgaard K. Bolus calculators. J Diabetes Sci Technol, 8(5):1035-1041, 2014.

[6] Walsh J., Roberts R., Bailey T. S., and Heinemann L. Bolus advisors: Sources of error, targets for improvement. Journal of Diabetes Science and Technology, 12(1):190-198, 2018.

[7] Grunberger G., Abelseth J., and Bailey T., et al. Consensus statement by the american association of clinical endocrinologists/american college of endocrinology insulin pump management task force. Endocrine Practice, 20(5):463-489, 2014. 